\title{
Network-based analysis of the molecular mechanisms of multiple myeloma and monoclonal gammopathy of undetermined significance
}

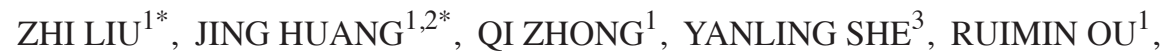

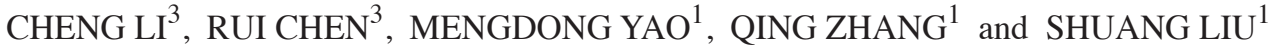 \\ ${ }^{1}$ Department of Hematology, Guangdong Second Provincial General Hospital, Guangzhou, Guangdong 510317; \\ ${ }^{2}$ Department of Hematology, The First Hospital of Kashgar District of Xinjiang, Kashgar, Xinjiang 844000; \\ ${ }^{3}$ Guangdong Traditional Medical and Sports Injury Rehabilitation Research Institute, \\ Guangdong Second Provincial General Hospital, Guangzhou, Guangdong 510317, P.R. China
}

Received March 17, 2017; Accepted June 15, 2017

DOI: $10.3892 / 01.2017 .6723$

\begin{abstract}
The present study aimed to reveal the molecular mechanisms of multiple myeloma (MM) and monoclonal gammopathy of undetermined significance (MGUS). This was a secondary study on microarray dataset GSE80608, downloaded from the Gene Expression Omnibus database, which included 10 control samples, 10 MGUS samples and $10 \mathrm{MM}$ samples. Differentially expressed genes (DEGs) were identified between control and MGUS samples, and between control and MM samples. A protein-protein interaction (PPI) network was built for studying the interactions between the DEGs. Kyoto Encyclopedia of Genes and Genomes pathway enrichment analysis was performed for the genes in a gene co-expression network. A microRNA (miRNA/miR)-gene network was built to the evaluate possible the miRNAs and genes involved in the diseases. The present study identified 136 common upregulated DEGs and 165 common downregulated DEGs between MM and MGUS. Pathway enrichment analysis of the genes in the gene co-expression network revealed that the complement and coagulation cascades pathway was significantly enriched for certain complement and coagulation-associated genes. Endothelin-1 (EDN1) was significantly enriched in the hypoxia inducible factor-1 (HIF-1) and tumor necrosis factor
\end{abstract}

Correspondence to: Dr Shuang Liu or Dr Qing Zhang, Department of Hematology, Guangdong Second Provincial General Hospital, 466 Xin Gang Zhong Road, Guangzhou, Guangdong 510317, P.R. China

E-mail: luuiS789@163.com

E-mail: zhqing@vip.163.com

${ }^{*}$ Contributed equally

Key words: multiple myeloma, monoclonal gammopathy of undetermined significance, pathway, gene co-expression network, microRNA signaling pathways. EDN1 was an important node in the PPI network, and a target gene of let-7e, let-7b and miR-19a in the miRNA-gene network. The results of the present study indicate that complement and coagulation-associated genes, the complement and coagulation cascades pathway, EDN1, let-7e, let-7b-5p, miR-19a, and the tumor necrosis factor and HIF-1 signaling pathways may all be implicated in MM and MGUS.

\section{Introduction}

Multiple myeloma (MM) is a cancer of the plasma cells within the bone marrow (BM), which caused $\sim 79,000$ mortalities in 2013 (1). It has been widely accepted that MM arises from monoclonal gammopathy of undetermined significance (MGUS), which is a pre-malignant condition that can be present for several years prior to the development of MM (2). As MM is incurable in the vast majority of patients (3), there is an urgent requirement for novel therapies. Intervening at the MGUS stage may aid to prevent the progression of MGUS to MM (4,5). Therefore, a thorough understanding of the molecular pathogenesis of MM and MGUS is urgently required to guide interventions that target the precursor state (MGUS) and MM itself.

Kubiczkova et al (6) revealed the identity of five microRNAs (miRNAs/miRs) that were deregulated in the circulating serum of patients with MM and MGUS, compared with that of healthy subjects. Shvartsur et al (7) suggested that Raf-1 kinase inhibitor protein-associated genes could be implicated in MM and MGUS using a bioinformatics approach. Amend et al (8) provided in vivo evidence that the absence of SAM domain, SH3 domain and nuclear localization signals 1 may be associated with genetic susceptibility to MGUS in mice. Furthermore, there is evidence that a dysregulated cyclin D/retinoblastoma signaling pathway is involved in the molecular pathogenesis of MM and MGUS $(9,10)$. The nuclear factor- $\kappa \mathrm{B}(\mathrm{NF}-\kappa \mathrm{B})$ signaling pathway serves an important role in the mechanism of MM pathogenesis (11). Despite these advances, the molecular mechanisms behind the transformation of MUGS into MM have not been fully elucidated. 
McNee et al (12) observed that the citrullination of histone $\mathrm{H} 3$ promotes interleukin-6 (IL-6) production by BM mesenchymal stem cells, resulting in pro-malignancy signaling in patients with MGUS and MM. However, the genes and pathways involved in MM and MGUS have not been fully delineated. Therefore, the present study reports a secondary analysis of the microarray dataset GSE80608 (12). Differentially expressed genes (DEGs) in MM, MGUS and control subjects were identified, and a protein-protein interaction (PPI) network was built to analyze the interactions between genes. A gene co-expression network was then built to analyze the co-expression of these DEGs in MGUS and MM. Kyoto Encyclopedia of Genes and Genomes (KEGG) pathway-enrichment analysis was performed to reveal the signaling pathways that may be involved in MGUS and MM. The data may provide indications of the genes and pathways that determine the progression of MGUS to MM.

\section{Materials and methods}

Microarray dataset preprocessing. The present study was a secondary study on the GSE80608 microarray dataset, obtained from the National Center for Biotechnology Information Gene Expression Omnibus (http://www.ncbi .nlm.nih.gov/geo/) (13) and based on the Affymetrix Human Exon 1.0 ST Array platform (Affymetrix; Thermo Fisher Scientific, Inc., Waltham, MA, USA). The dataset consisted of 10 control BM samples, 10 BM samples from the iliac crest of 10 patients with MGUS and 10 BM samples from the iliac crest of 10 patients with MM.

With the aid of oligo software (version 5.1; National Biosciences, Inc., Plymouth, Minn., USA), the raw data were subjected to data preprocessing, including background correction, data normalization and calculation of probe expression values. By using huex10sttranscriptcluster.db (14) and annotate (15) software in R (16), each probe was mapped to its corresponding gene symbol.

DEGs screening. DEGs were screened between MGUS and control, and between MM and control samples, using the LIMMA package in $\mathrm{R}$ (17). A strict cutoff was set at a false discovery rate $<0.05$ and fold change $\left(\log _{2} \mathrm{FCl}\right) \geq 1.5$.

Venn diagram analysis and KEGG pathway enrichment analysis. DEGs in MGUS or MM were analyzed using a Venn diagram (18), which revealed the number of the common upregulated DEGs, common downregulated DEGs and contra-regulated DEGs between MGUS and MM in a Venn diagram.

KEGG pathway enrichment (19) analysis was performed for the identified DEGs using DAVID software (20). KEGG pathways with a gene count $\geq 2$ and $\mathrm{P}<0.05$ were considered to indicate a statistically significant difference.

Analysis of a PPI network. A PPI network (PPI score=0.7) was constructed with the identified DEGs using the STRING online tool (21) to analyze the interactions between the DEGs. In the PPI network, a node denoted a gene and a link between two nodes denoted the interaction between the two genes. The number of interactions between one gene and other genes in the network was represented by the 'degree' value. The topological

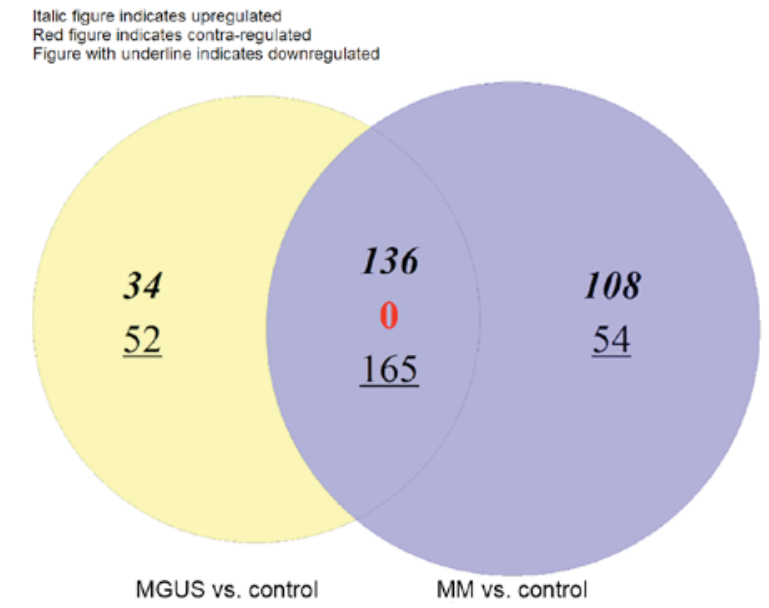

Figure 1. Venn diagram of differently expressed genes in MM and MGUS. MM, multiple myeloma; MGUS, monoclonal gammopathy of undetermined significance.

parameters of the nodes in the PPI network were analyzed using the Cytoscape CytoNCA application (22), and included degree, betweenness (23), closeness (24) and subgraph (25).

Gene co-expression analysis. A gene co-expression network was built to analyze the co-expression of the DEGs in MGUS and MM. The co-expression coefficient between two genes was calculated based on the Pearson's correlation coefficient. The P-value was calculated using the Z-score (26). Significant co-expressed gene pairs were determined to have a co-expression coefficient $>0.85$ and $\mathrm{P}<0.05$. KEGG pathway enrichment analysis was then conducted for the co-expressed genes.

Analysis of disease-related miRNA and target genes. MM-associated miRNAs were downloaded from the miR2Disease database (27), which included 17 miRNAs that were associated with MM. On the basis of these miRNAs, target genes were predicted using miRwalk2.0 (28). The miRNA-target gene interaction pairs were obtained from the validated target module in miRwalk2.0, which had been validated previously (28). Finally, the differentially expressed target genes were selected.

\section{Results}

Identification and Venn diagram analysis of DEGs. The present study identified 387 DEGs between MGUS and control samples, and 463 DEGs between MM and control samples. Venn diagram analysis revealed that there were 136 common upregulated DEGs, 165 common downregulated DEGs and no contra-regulated DEGs between MGUS and MM (Fig. 1). MGUS had 34 specific upregulated DEGs and 52 specific downregulated DEGs, whereas MM had 108 specific upregulated DEGs and 54 specific downregulated DEGs. A heat map revealed that DEG expression in the control samples was markedly different from that in MM and MGUS samples (Fig. 2).

KEGG pathway enrichment analysis. KEGG pathway enrichment analysis (Table I) revealed that the common upregulated DEGs were significantly enriched in five pathways, including 


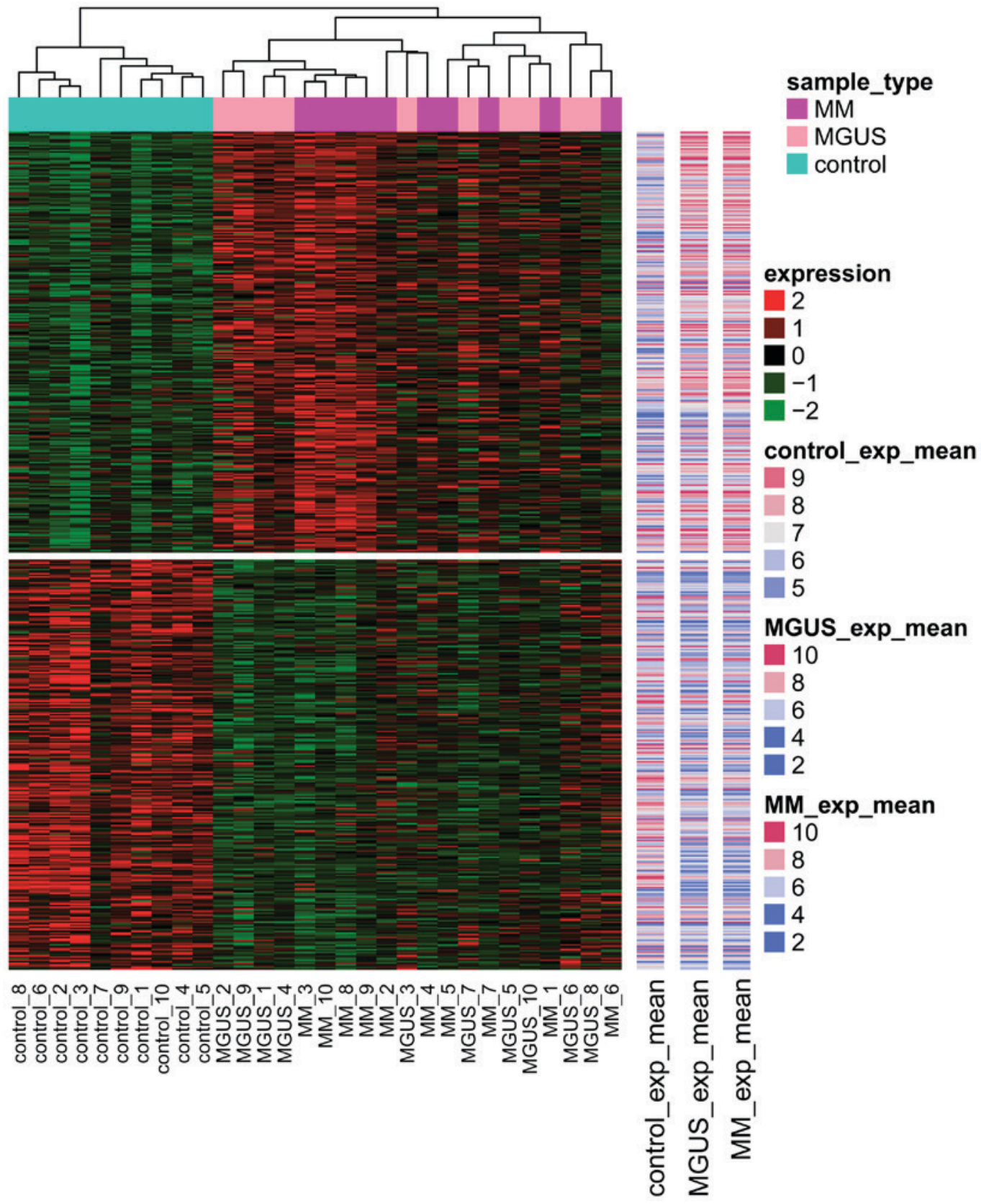

Figure 2. Heat map of differently expressed genes. MM, multiple myeloma; MGUS, monoclonal gammopathy of undetermined significance; exp, expression.

the Rap1 signaling pathway and the regulation of actin cytoskeleton pathway. The common downregulated DEGs were significantly associated with the complement and coagulation cascades, including cluster of differentiation 55 (CD55), thrombomodulin (THBD), mannan-binding lectin serine protease 1 (MASP1), complement factor B (CFB), coagulation factor III (F3), complement component $1(\mathrm{C} 1) \mathrm{R}, \mathrm{C} 1 \mathrm{~S}$ and coagulation factor II receptor (F2R). Furthermore, common downregulated DEGs were significantly associated with axon guidance, ATP-binding cassette transporters, Staphylococcus aureus infection response and steroid hormone biosynthesis pathways. MM-specific upregulated DEGs were significantly associated with the regulation of actin cytoskeleton pathway.

PPI network. A PPI network was built with the DEGs in MM and MGUS (Fig. 3). The network included 233 nodes and 435 gene pairs. Of these genes, 39 were MGUS-specific, 59 were MM-specific and 125 were common between MGUS and MM.

In the PPI network, the top 20 genes (nodes) were selected, based on their values of degree, betweenness, closeness and subgraph, to be the important nodes in the PPI network (Table II). Of the top 20 genes, endothelin 1 (EDN1), C-X-C motif chemokine ligand 12 , adenylate cyclase 4 , matrix metallopeptidase 1, insulin like growth factor 1 and Finkel-Biskis-Jinkins osteosarcoma oncogene were common between MGUS and MM.

Analysis of a gene co-expression network. The gene co-expression network included 312 co-expressed gene pairs, 186 MM- or MGUS-specific genes, and 141 genes common to MM and MGUS. The co-expressed genes exhibited consistent changes in MM and MGUS (Fig. 4). These co-expressed genes 
Table I. Significant Kyoto Encyclopedia of Genes and Genomes pathways of DEGs.

\begin{tabular}{|c|c|c|c|}
\hline DEGs & Pathway & P-value & Genes \\
\hline \multirow[t]{5}{*}{$\begin{array}{l}\text { Common upregulated } \\
\text { DEGs }\end{array}$} & hsa04360: Axon guidance & $8.43 \times 10^{-4}$ & $\begin{array}{l}\text { PLXNA3, SEMA7A, MET, NTN4, SEMA3A, } \\
\text { CXCL12, EPHA3 }\end{array}$ \\
\hline & $\begin{array}{l}\text { hsa04514: Cell adhesion } \\
\text { molecules }\end{array}$ & $8.17 \times 10^{-3}$ & $\begin{array}{l}\text { NRXN3, ICAM2, CD4, ITGB2, NECTIN3, } \\
\text { PDCD1LG2 }\end{array}$ \\
\hline & $\begin{array}{l}\text { hsa05200: Pathways in } \\
\text { cancer }\end{array}$ & $2.16 \times 10^{-2}$ & $\begin{array}{l}\text { FGF5, PLCB4, PGF, SLC2A1, MET, BDKRB1, } \\
\text { ITGA3, FGF1, CXCL12 }\end{array}$ \\
\hline & $\begin{array}{l}\text { hsa04015: Rap1 signaling } \\
\text { pathway }\end{array}$ & $3.75 \times 10^{-2}$ & FGF5, PLCB4, PGF, MET, ITGB2, FGF1 \\
\hline & $\begin{array}{l}\text { hsa04810: Regulation of } \\
\text { actin cytoskeleton }\end{array}$ & $3.82 \times 10^{-2}$ & FGF5, SCIN, BDKRB1, ITGB2, ITGA3, FGF1 \\
\hline \multirow[t]{5}{*}{$\begin{array}{l}\text { Common downregulated } \\
\text { DEGs }\end{array}$} & $\begin{array}{l}\text { hsa04610: Complement and } \\
\text { coagulation cascades }\end{array}$ & $7.15 \times 10^{-6}$ & CD55, THBD, MASP1, CFB, F3, C1R, C1S, F2R \\
\hline & hsa04360: Axon guidance & $1.09 \times 10^{-2}$ & $\begin{array}{l}\text { EPHA4, SEMA6D, NTNG1, EFNA5, UNC5D, } \\
\text { SLIT3 }\end{array}$ \\
\hline & hsa02010: ABC transporters & $1.11 \times 10^{-2}$ & ABCA8, АBCC9, АBCA9, АBCA6 \\
\hline & $\begin{array}{l}\text { hsa05150: Staphylococcus } \\
\text { aureus infection }\end{array}$ & $1.65 \times 10^{-2}$ & MASP1, CFB, C1R, C1S \\
\hline & $\begin{array}{l}\text { hsa00140: Steroid hormone } \\
\text { biosynthesis }\end{array}$ & $2.32 \times 10^{-2}$ & AKR1C3, AKR1C2, HSD11B1, AKR1C1 \\
\hline $\begin{array}{l}\text { MM-specific } \\
\text { upregulated DEGs }\end{array}$ & $\begin{array}{l}\text { hsa04810: Regulation of } \\
\text { actin cytoskeleton }\end{array}$ & $4.79 \times 10^{-2}$ & RAC2, MRAS, ITGA8, DIAPH3, ITGA7 \\
\hline \multirow[t]{2}{*}{$\begin{array}{l}\text { MGUS-specific } \\
\text { downregulated DEGs }\end{array}$} & $\begin{array}{l}\text { hsa05410: Hypertrophic } \\
\text { cardiomyopathy }\end{array}$ & $1.91 \times 10^{-2}$ & SGCD, IGF1, CACNA2D3 \\
\hline & $\begin{array}{l}\text { hsa05414: Dilated } \\
\text { cardiomyopathy }\end{array}$ & $2.20 \times 10^{-2}$ & SGCD, IGF1, CACNA2D3 \\
\hline
\end{tabular}

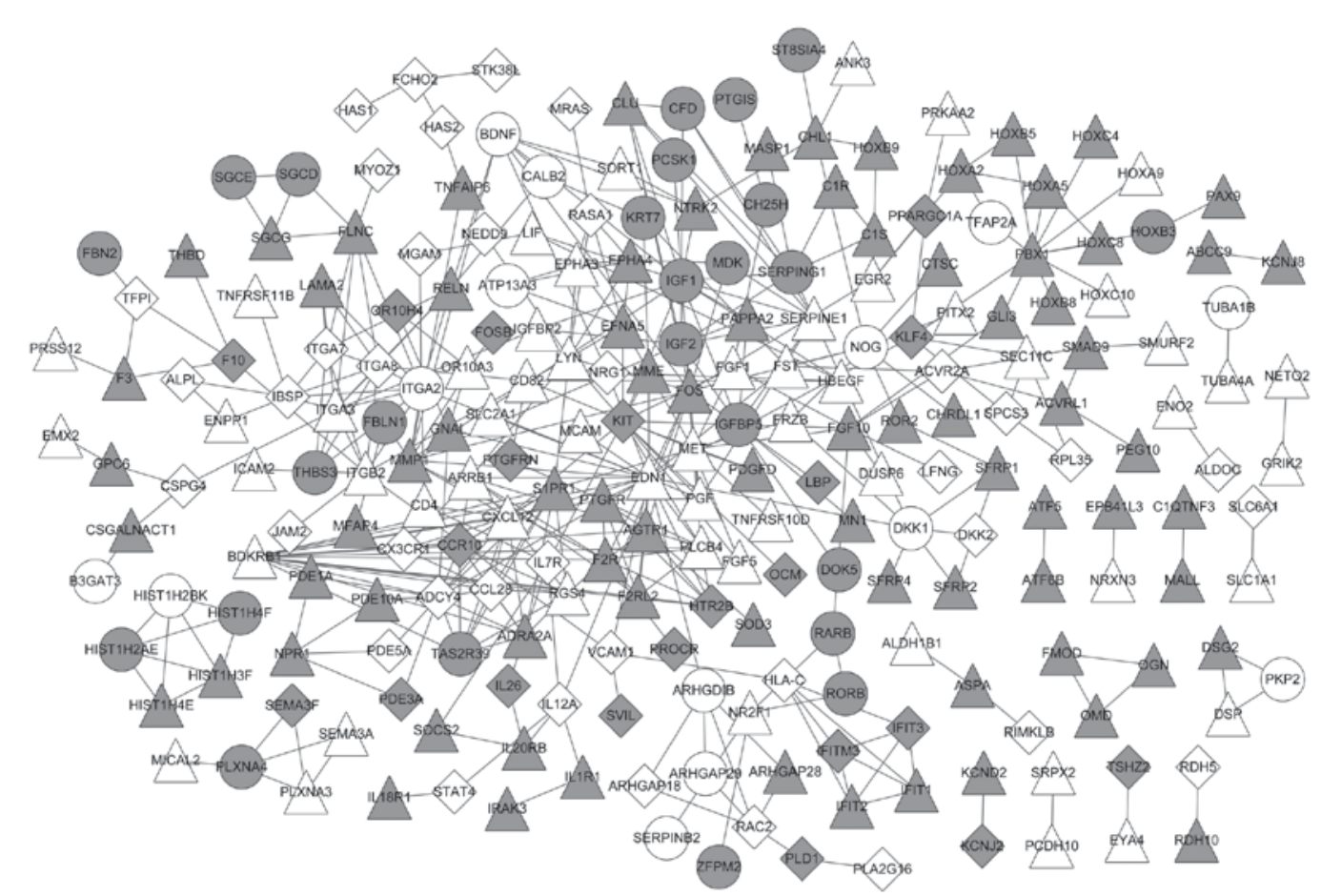

Figure 3. Protein-protein interaction network. Grey nodes, downregulated genes; white nodes, upregulated genes; round nodes, MGUS-specific genes; diamond nodes, MM-specific genes; triangular nodes, common genes between MGUS and MM. A link between two nodes represents an interaction between two genes. MGUS, monoclonal gammopathy of undetermined significance; MM, multiple myeloma. 
Table II. Degree, betweenness, closeness and subgraph of the top 20 nodes in the protein-protein interaction network.

\begin{tabular}{|c|c|c|c|c|}
\hline Node, gene & Subgraph & Degree & Betweenness & Closeness \\
\hline BDKRB1 & $1,515.014801$ & 15 & & $2.18 \times 10^{-2}$ \\
\hline EDN1 & $1,337.5355$ & 24 & $14,298.21$ & $2.20 \times 10^{-2}$ \\
\hline CXCL12 & $1,014.57958$ & 14 & $1,799.90$ & $2.18 \times 10^{-2}$ \\
\hline ADCY4 & 691.12317 & & & \\
\hline F2R & 687.05615 & 11 & & $2.18 \times 10^{-2}$ \\
\hline CCR 10 & 641.9886599 & 9 & & \\
\hline S1PR1 & 633.8678 & 10 & & \\
\hline CCL28 & 597.2848528 & 9 & & \\
\hline AGTR1 & 580.683 & 10 & & $2.18 \times 10^{-2}$ \\
\hline ADRA2A & 559.71972 & & & \\
\hline TAS2R39 & 559.718772 & & & \\
\hline RGS4 & 542.425323 & & & \\
\hline F2RL2 & 511.2666327 & & & \\
\hline PLCB4 & 510.37097 & 9 & & $2.18 \times 10^{-2}$ \\
\hline PTGFR & 486.30338 & & & \\
\hline HTR2B & 432.8887689 & & & \\
\hline ARRB1 & 363.97263 & & & \\
\hline MMP1 & 248.22731 & 11 & $4,609.06$ & $2.19 \times 10^{-2}$ \\
\hline IGF1 & 233.1976220 & 17 & $4,473.53$ & \\
\hline FOS & 213.7347773 & 13 & $6,338.76$ & $2.19 \times 10^{-2}$ \\
\hline ADCY4 & & 13 & $2,133.74$ & $2.18 \times 10^{-2}$ \\
\hline KIT & & 13 & $4,026.67$ & $2.18 \times 10^{-2}$ \\
\hline ITGA2 & & 12 & $5,403.44$ & $2.19 \times 10^{-2}$ \\
\hline MET & & 12 & $3,152.53$ & $2.18 \times 10^{-2}$ \\
\hline PBX1 & & 11 & $3,496.13$ & \\
\hline ITGA8 & & 10 & & \\
\hline LYN & & 10 & $3,282.67$ & $2.18 \times 10^{-2}$ \\
\hline ITGA3 & & 10 & $2,372.76$ & \\
\hline KLF4 & & & $4,272.19$ & \\
\hline ITGB2 & & & $3,974.00$ & \\
\hline FGF10 & & & $3,904.25$ & \\
\hline VCAM1 & & & $2,679.60$ & \\
\hline NOG & & & $2,237.87$ & \\
\hline ARHGDIB & & & $2,199.00$ & \\
\hline IL12A & & & $1,979.33$ & \\
\hline F10 & & & $1,848.00$ & \\
\hline FST & & & & $2.17 \times 10^{-2}$ \\
\hline BDNF & & & & $2.18 \times 10^{-2}$ \\
\hline ARRB1 & & & & $2.18 \times 10^{-2}$ \\
\hline MME & & & & $2.18 \times 10^{-2}$ \\
\hline DKK1 & & & & $2.18 \times 10^{-2}$ \\
\hline PGF & & & & $2.19 \times 10^{-2}$ \\
\hline IGF1 & & & & $2.19 \times 10^{-2}$ \\
\hline
\end{tabular}

were significantly associated with the complement and coagulation cascades pathway, tumor necrosis factor (TNF) signaling pathway, $S$. aureus infection pathway, hypoxia-inducible factor-1 (HIF-1) signaling pathway and pathways in cancer (Table III).

Analysis of a miRNAs-gene network. A total of $16 \mathrm{MM}$-associated miRNAs were obtained from the miR2Disease database: miR-99b, miR-93, miR-561, miR-342, miR-335, miR-32, miR-25, miR-19b, miR-19a, miR-181b, miR-181a,
miR-140-3p, miR-125a-5p, miR-106b, let-7e and let-7b. Fig. 5 shows a miRNA-gene network, featuring the associations between the 16 miRNAs and their target genes.

\section{Discussion}

MGUS is premalignant plasma-cell proliferative condition that consistently precedes MM (29). The current study identified that common downregulated DEGs, including CD55, THBD, MASP1, CFB, F3, C1R, C1S and F2R, were significantly 


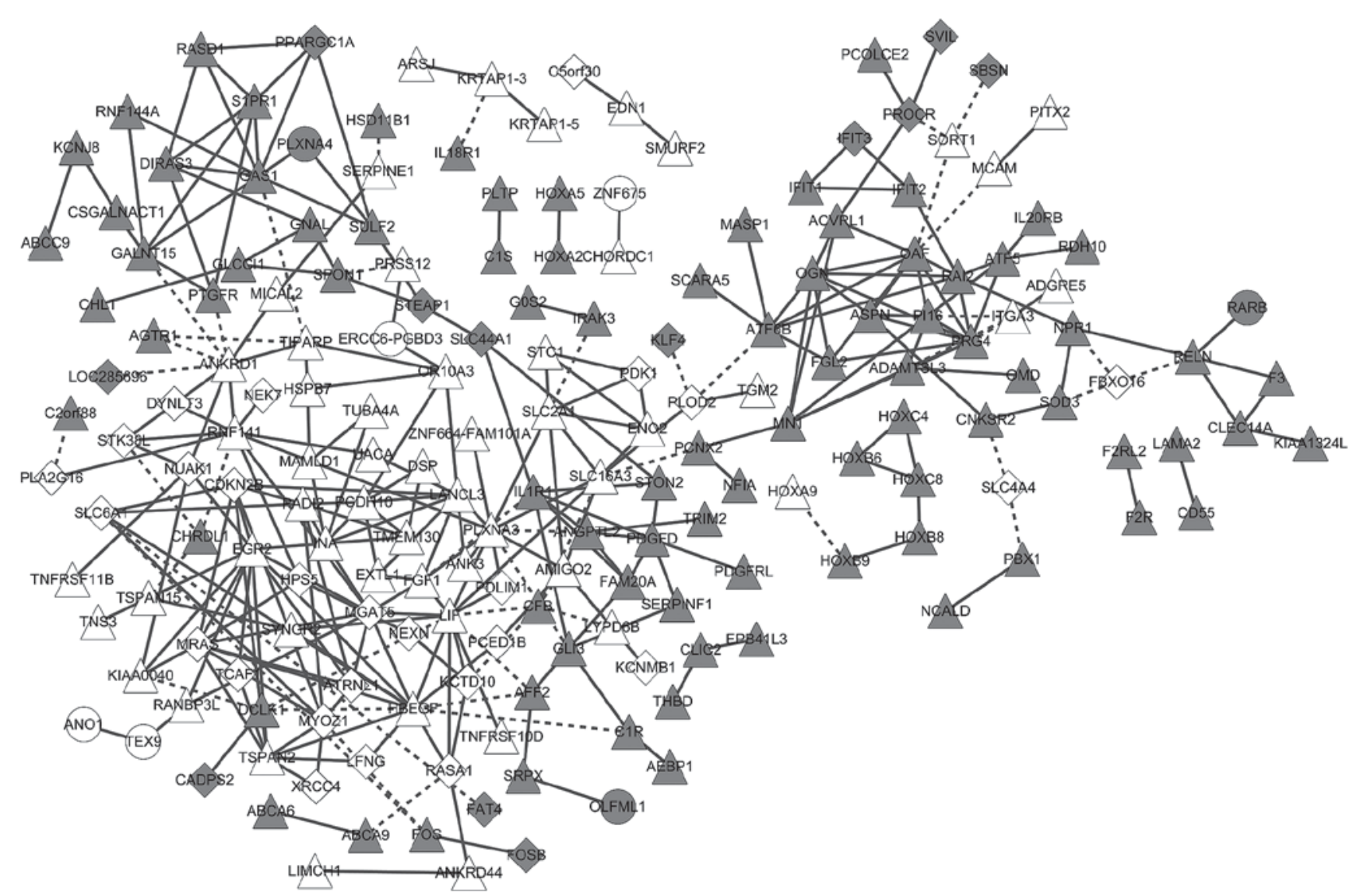

Figure 4. Gene co-expression network. Grey nodes, downregulated genes; white nodes, upregulated genes; round nodes, MGUS-specific genes; diamond nodes, MM-specific genes; triangular nodes, common genes between MGUS and MM. Solid lines indicate positive correlations, dotted lines indicate negative correlations. MGUS, monoclonal gammopathy of undetermined significance; MM, multiple myeloma.

enriched in complement and coagulation cascades pathway. CD55 encodes complement decay-accelerating factor, which regulates the complement system on the cell surface (30). THBD encodes thrombomodulin, which participates in the anticoagulant pathway. MASP1 is an enzyme involved in the lectin pathway of the complement system (31). CFB, F3, C1R, $\mathrm{C} 1 \mathrm{~S}$ and $\mathrm{F} 2 \mathrm{R}$ are components of the complement or coagulation system (32). Crowely et al (33) suggested that patients with MGUS have an intermediate coagulation profile, between that of patients with myeloma and that of healthy controls. There is evidence that MM is associated with an increased risk of venous thromboembolism (34). These findings indicate that these downregulated complement and coagulation genes may be involved in the development of MM and MGUS through the regulation of the complement and coagulation cascade pathways.

HIFs are transcription factors that respond to hypoxia; the HIF signaling pathway mediates the effects of hypoxia on the cell (35). It has been suggested that a hypoxic BM environment serves a role in the pathogenesis of MM $(36,37)$. Azab et al (38) provided evidence that hypoxia promotes the dissemination of MM. In concordance with these findings, the current study noticed that the HIF-1 signaling pathway was significantly enriched for co-expressed genes. This enrichment indicates that the HIF-1 signaling pathway may have a role in the development of MM and MGUS. Furthermore, suppression of HIF-1 decreases the expression of the anti-apoptotic protein survivin, strengthening the sensitivity of MM cells to melphalan (39). HIF-1 inhibition may be a promising therapeutic strategy for treating MM and premalignant MGUS.

Lee et al (40) reported that TNF expression is significantly increased in patients with active MM and regulates IL-6 production. Previous studies have demonstrated that the NF- $\kappa B$ pathway, which is downstream of TNF, is involved in MM $(11,41)$. In the present study, the TNF signaling pathway was significantly enriched by co-expressed genes. EDN1, a common DEG between MGUS and MM and an important node in the PPI network, was significantly enriched in the TNF and HIF-1 signaling pathways. EDN1 is a potent vasoconstrictor produced by vascular endothelial cells, whose expression is stimulated by hypoxia (42). These findings indicate that END1 could serve an important role in premalignant MGUS and MM by influencing the TNF and HIF-1 signaling pathways.

The present study additionally investigated a miRNA-gene regulatory network. In the miRNA-gene regulatory network, END1 was regulated by let-7e-5p, let-7b-5p, and miR-19a-5p, suggesting that the role of EDN1 in premalignant MGUS and MM may be affected by these miRNAs. Similarly, Kubiczkova et al (6) revealed that circulating serum levels of let-7e are deregulated in MM and MGUS compared with those in healthy subjects.

The present study has certain limitations. Firstly, the study samples are limited. It is a secondary study on the microarray dataset GSE80608, meaning that the number of samples in 
Table III. Significantly enriched pathways of genes in the gene co-expression network.

\begin{tabular}{lll}
\hline Pathway & P-value & \\
\hline hsa04610: Complement and coagulation cascades & $8.11 \times 10^{-7}$ & CD55, THBD, MASP1, CFB, F3, SERPINE1, C1R, \\
& & C1S, F2R \\
hsa05150: Staphylococcus aureus infection & 0.019029 & MASP1, CFB, C1R, C1S \\
hsa04066: HIF-1 signaling pathway & 0.023618 & PDK1, EDN1, SLC2A1, SERPINE1, ENO2 \\
hsa04668: TNF signaling pathway & 0.029493 & LIF, IL18R1, FOS, EDN1, ATF6B \\
hsa05200: Pathways in cancer & 0.030231 & LAMA2, AGTR1, FOS, CDKN2B, SLC2A1, ITGA3, \\
& & RARB, FGF1, GLI3, F2R
\end{tabular}

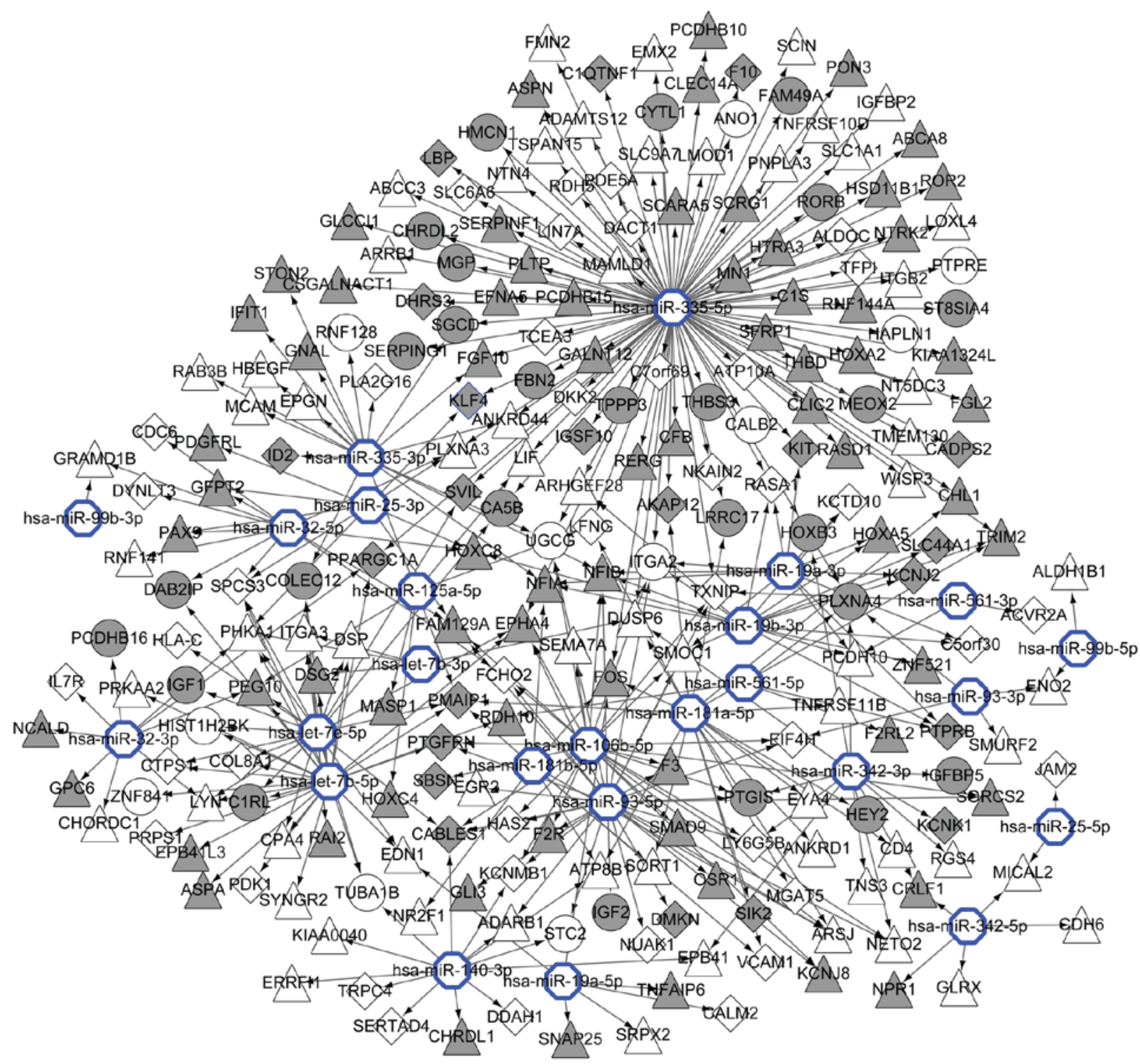

Figure 5. miRNA-gene network. Blue hexagonal nodes represent miRNAs; grey nodes, downregulated genes; white nodes, upregulated genes; round nodes, MGUS-specific genes; diamond nodes, MM-specific genes; triangular nodes, common genes between MGUS and MM. Connecting arrows indicate regulatory interactions between miRNAs and target genes. MGUS, monoclonal gammopathy of undetermined significance; MM, multiple myeloma; miRNA, microRNA .

the study cannot be increased. Secondly, the study only used bioinformatics approaches, meaning that experimental data are necessary to validate these findings in further studies.
The present study suggests that the complement and coagulation-associated genes EDN1, let-7e, let-7b, miR-19a, along with the complement and coagulation, and the TNF and 
HIF-1 signaling pathways, may be involved in the molecular mechanism of MM and MGUS pathogenesis. Further experiments are warranted to verify the findings of the current study.

\section{Acknowledgements}

The present study was supported by grants from the National Natural Science Foundation of China (no. 81400168); the Guangzhou Health Care and Cooperative Innovation Major Project (no. 201400000003-1); the Science and Technology Planning Project of Guangdong Province, China (no. 2014A020209047 and 2015A020210068); the Foundation of Guangdong Traditional Chinese Medicine (no. 20131104); the Foundation of Guangdong Medicine (no. A2013128 and A2017266); the Foundation of Technological Support of Xinjiang Uygur Autonomous Region (no. 201491185); and the Foundation of Guangdong Second Provincial General Hospital (grant nos. YY2014-002, YQ2015-004/005/012/016, 2016-011/013 and 2017001).

\section{References}

1. GBD 2013 Mortality and Causes of Death Collaborators: Global, regional, and national levels of age-sex specific all-cause and cause-specific mortality for 240 causes of death, 1990-2013: A systematic analysis for the Global Burden of Disease Study. Lancet 385: 117-171, 2015.

2. Landgren O, Kyle RA, Pfeiffer RM, Katzmann JA, Caporaso NE, Hayes RB, Dispenzieri A, Kumar S, Clark RJ, Baris D, et al: Monoclonal gammopathy of undetermined significance (MGUS) consistently precedes multiple myeloma: A prospective study. Blood 113: 5412-5417, 2009.

3. Berenson JR, Matous J, Swift RA, Mapes R, Morrison B and Yeh HS: A phase I/II study of arsenic trioxide/bortezomib/ascorbic acid combination therapy for the treatment of relapsed or refractory multiple myeloma. Clin Cancer Res 13: 1762-1768, 2007.

4. Agarwal A and Ghobrial IM: Monoclonal gammopathy of undetermined significance and smoldering multiple myeloma: A review of the current understanding of epidemiology, biology, risk stratification, and management of myeloma precursor disease. Clin Cancer Res 19: 985-994, 2013.

5. Landgren O: Monoclonal gammopathy of undetermined significance and smoldering multiple myeloma: Biological insights and early treatment strategies. Hematology Am Soc Hematol Educ Program 2013: 478-487, 2013.

6. Kubiczkova L, Kryukov F, Slaby O, Dementyeva E, Jarkovsky J, Nekvindova J, Radova L, Greslikova H, Kuglik P, Vetesnikova E, et al: Circulating serum microRNAs as novel diagnostic and prognostic biomarkers for multiple myeloma and monoclonal gammopathy of undetermined significance. Haematologica 99: 511-518, 2014.

7. Shvartsur A, Chatterjee D, Chen H, Berenson J, Garban H and Bonavida B: A bioinformatics approach revealed a differential expression of RKIP-related genes in pre-multiple myeloma and multiple myeloma: Clinical implications. Blood 126: 5309, 2015.

8. Amend S, Liang C, Serie D, Vachon CM, Lu L, Vij R, Colditz GA Weilbaecher KN and Tomasson MH: Deletion of samsn1 underlies genetic susceptibility to Monoclonal Gammopathy of undetermined significance (MGUS) in mice. Blood 122: 397, 2013.

9. Kuehl WM and Bergsagel PL: Molecular pathogenesis of multiple myeloma and its premalignant precursor. J Clin Invest 122 3456-3463, 2012.

10. Bergsagel PL, Kuehl WM, Zhan F, Sawyer J, Barlogie B and Jr SJ: Cyclin D dysregulation: An early and unifying pathogenic event in multiple myeloma. Blood 106: 296-303, 2005.

11. Annunziata CM, Davis RE, Demchenko Y, Bellamy W, Gabrea A, Zhan F, Lenz G, Hanamura I, Wright G, Xiao W, et al: Frequent engagement of the classical and alternative NF-kappaB pathways by diverse genetic abnormalities in multiple myeloma. Cancer Cell 12: 115-130, 2007.
12. McNee G, Eales KL, Wei W, Williams DS, Barkhuizen A, Bartlett DB, Essex S, Anandram S, Filer A, Moss PA, et al: Citrullination of histone $\mathrm{H} 3$ drives IL- 6 production by bone marrow mesenchymal stem cells in MGUS and multiple myeloma. Leukemia 31: 373-381, 2017.

13. Clough $\mathrm{E}$ and Barrett T: The gene expression omnibus database. Methods Mol Biol 1418: 93-110, 2016.

14. MacDonald JW: Huex10sttranscriptcluster. db: Affymetrix huex10 annotation data (chip huex10sttranscriptcluster). $\mathrm{R}$ package version 8.2.0, 2017

15. Gentleman R: Annotate: Annotation for microarrays. R package version 1.54.0, 2017.

16. R Development Core Team: R: A language and environment for statistical computing. R Foundation for Statistical Computing, Vienna, Austria, 2017.

17. Smyth GK: In: Gentleman R, Carey V, Dudoit S, Irizarry R and Huber W, eds. Limma: Linear models for microarray data. Bioinformatics and Computational Biology Solutions Using R and Bioconductor, Springer New York: pp397-420, 2005.

18. Chen $\mathrm{H}$ and Boutros PC: VennDiagram: A package for the generation of highly-customizable Venn and Euler diagrams in R. Bmc Bioinformatics 12: 35, 2011.

19. Kanehisa M, Goto S, Sato Y, Furumichi M and Tanabe M: KEGG for Integration and Interpretation of large-scale molecular data sets. Nucleic Acids Res 40 (Database issue): D109-D114, 2012.

20. Dennis G, Sherman BT, Hosack DA, Yang J, Gao W, Lane HC and Lempicki RA: DAVID: Database for annotation, visualization, and integrated discovery. Genome Biol 4: P3, 2003.

21. Jensen LJ, Kuhn M, Stark M, Chaffron S, Creevey C, Muller J, Doerks T, Julien P, Roth A, Simonovic M, et al: STRING 8-a global view on proteins and their functional interactions in 630 organisms. Nucleic Acids Res 37 (Database issue): D412-D416, 2009.

22. Tang Y, Li M, Wang J, Pan Y and Wu FX: CytoNCA: A cytoscape plugin for centrality analysis and evaluation of protein interaction networks. Biosystems 127: 67-72, 2015.

23. Goh KI, Oh E, Kahng B and Kim D: Betweenness centrality correlation in social networks. Phys Rev E Stat Nonlin Soft Matter Phys 67: 017101, 2003.

24. Du Y, Gao C, Chen X, Hu Y, Sadiq R and Deng Y: A new closeness centrality measure via effective distance in complex networks. Chaos 25: 033112, 2015.

25. Estrada E and Rodríguezvelázquez JA: Subgraph centrality in complex networks. Phys Rev E Stat Nonlin Soft Matter Phys 71: 056103, 2005.

26. Liang M, Zhang F, Jin G and Zhu J: FastGCN: A GPU accelerated tool for fast gene co-expression networks. PloS One 10: $\mathrm{e} 0116776,2015$.

27. Jiang Q, Wang Y, Hao Y, Juan L, Teng M, Zhang X, Li M, Wang $\mathrm{G}$ and Liu Y: miR2Disease: A manually curated database for microRNA deregulation in human disease. Nucleic Acids Res 37 (Suppl 1): D98-D104, 2009.

28. Dweep H and Gretz N: miRWalk2.0: A comprehensive atlas of microRNA-target interactions. Nat Methods 12: 697, 2015.

29. Weiss BM, Abadie J, Verma P, Howard RS and Kuehl WM: A monoclonal gammopathy precedes multiple myeloma in most patients. Blood 113: 5418-5422, 2009.

30. Spendlove I, Ramage JM, Bradley R, Harris C and Durrant LG: Complement decay accelerating factor (DAF)/CD55 in cancer. Cancer Immunol Immunother 55: 987-995, 2006.

31. Matsushita M, Thiel S, Jensenius JC, Terai I and Fujita T: Proteolytic activities of two types of mannose-binding lectin-associated serine protease. J Immunol 165: 2637-2642, 2000.

32. Markiewski MM, Nilsson B, Ekdahl KN, Mollnes TE and Lambris JD: Complement and coagulation: Strangers or partners in crime? Trends Immunol 28: 184-192, 2007.

33. Crowely MP, Quinn S, Coleman E, Eustace JA, Gilligan OM and O'Shea SI: Differing coagulation profiles of patients with monoclonal gammopathy of undetermined significance and multiple myeloma. J Thromb Thrombolysis 39: 245-249, 2015.

34. De Stefano V, Za T and Rossi E: Venous thromboembolism in multiple myeloma. Semin Thromb Hemost 40: 338-347, 2014

35. Kaelin WG Jr and Ratcliffe PJ: Oxygen sensing by metazoans: The central role of the HIF hydroxylase pathway. Mol Cell 30: 393-402, 2008.

36. Martin SK, Diamond P, Gronthos S, Peet DJ and Zannettino AC: The emerging role of hypoxia, HIF-1 and HIF-2 in multiple myeloma. Leukemia 25: 1533-1542, 2011. 
37. Asosingh K, De Raeve H, de Ridder M, Storme GA, Willems A, Van Riet I, Van Camp B and Vanderkerken K: Role of the hypoxic bone marrow microenvironment in 5T2 MM murine myeloma tumor progression. Haematologica 90: 810-817, 2005.

38. Azab AK, Hu J, Quang P, Azab F, Pitsillides C, Awwad R, Thompson B, Maiso P, Sun JD, Hart CP, et al: Hypoxia promotes dissemination of multiple myeloma through acquisition of epithelial to mesenchymal transition-like features. Blood 119 : 5782-5794, 2012.

39. Hu Y, Kirito K, Yoshida K, Mitsumori T, Nakajima K, Nozaki Y, Hamanaka S, Nagashima T, Kunitama M, Sakoe K and Komatsu N: Inhibition of hypoxia-inducible factor-1 function enhances the sensitivity of multiple myeloma cells to melphalan. Mol Cancer Ther 8: 2329-2338, 2009.

40. Lee C, Oh JI, Park J, Choi JH, Bae EK, Lee HJ, Jung WJ, Lee DS Ahn KS and Yoon SS: TNF $\alpha$ mediated IL-6 secretion is regulated by JAK/STAT pathway but Not by MEK phosphorylation and AKT phosphorylation in U266 multiple myeloma cells. Biomed Res Int 2013: 580135, 2013.
41. Mitsiades CS, Mitsiades N,Poulaki V,Schlossman R, Akiyama M, Chauhan D, Hideshima T, Treon SP, Munshi NC, Richardson PG and Anderson KC: Activation of NF-kappaB and upregulation of intracellular anti-apoptotic proteins via the IGF-1/Akt signaling in human multiple myeloma cells: Therapeutic implications. Oncogene 21: 5673-5683, 2002

42. Elton TS, Oparil S, Taylor GR, Hicks PH, Yang RH, Jin H and Chen YF: Normobaric hypoxia stimulates endothelin-1 gene expression in the rat. Am J Physiol 263: R1260-1264, 1992. 